\title{
Enhanced layerwise model for laminates with imperfect interfaces - Part 2: Experimental validation and failure prediction
}

\author{
Mendoza-Navarro Luis-Ernesto ${ }^{1}$, Diaz-Diaz Alberto ${ }^{1 *}$, Caron Jean-François ${ }^{2}$, \\ Chataigner Sylvain ${ }^{3}$ \\ ${ }^{1}$ Centro de Investigación en Materiales Avanzados, Miguel de Cervantes 120, C.I. \\ Chihuahua, 31109, Chihuahua Mexico. \\ ${ }^{2}$ Université Paris-Est, Institut Navier, LAMI-Ecole des Ponts, 6-8 Av. Blaise Pascal, \\ Marne La Vallée, 77455, France. \\ ${ }^{3}$ LUNAM Université. IFSTTAR Centre de Nantes, SOA. Bouguenais, F-443441 \\ France. \\ * Corresponding author. E-mail: alberto.diaz@cimav.edu.mx
}

Phone: (52-614)439-48-02. Fax: (52-614)439-11-30

\begin{abstract}
In this paper, the layerwise model for laminates with imperfect interfaces and enhanced in Part 1 of this work is confronted with experimental results. The model calculations are validated by comparing its sliding predictions in double lap adhesive joints to the sliding measurements performed in a previous paper. The model predictions agree with the experimental results. Finally, the model is applied to the failure analysis in double lap joints and T-peel joints exhibiting adherend, adhesive and cohesive failures. The model calculations and pertinent failure criteria provide accurate predictions and may become a helpful tool suitable to the design of adhesive joints.
\end{abstract}

Keywords: Interfaces; Adhesive Joints; Failure; Plasticity; Layerwise Model 


\section{Introduction}

An optimized structural design requires operational models to calculate stresses and predict strength. For laminated structures and adhesive joints with a plate or shell topology, several 2D models have been developed [1-4] to calculate stresses and displacements. Interfaces play an important role in the strength of laminates and adhesive joints [5-10]. Consequently, an accurate strength prediction must involve a calculation of interfacial stresses.

Layerwise models are suitable to the analysis and accurate evaluation of interfacial stresses and edge effects on these stresses. The unified formulation proposed by Carrera et al $[11,12]$ is a powerful tool which may be applied to build layerwise models. In these models, an approximation of displacements or stresses and displacements through the thickness of each layer is proposed. The Reissner's variational method [13] or the principle of virtual displacements is commonly used to obtain the constitutive equations. Another type of layerwise model is the local model developed by Pagano [14]. The local model starts with a stress approximation through the thickness of each layer and applies the Reissner's variational method to obtain the equations of the model. The stress field selection verifies the continuity conditions across the interfaces of the multilayer. The key point which is not often highlighted is that no displacement approximation is made despite the use of a mixed formulation. The Hellinger-Reissner functional helps to identify 2D generalized displacements, energetically associated to the generalized forces which derived to the stress approximation, but no constraint conditions on the 3D displacement fields are made. "Note that we refrain from assuming the form of the displacement field in accordance with the objectionable features of that approach" ([14], p.389). These considerations lead to a less constraint model than those where both displacement and stress components are approximated. Moreover the study of stress controlled phenomena could be more natural and more convenient with a direct description of stress fields. More recently, Pagano's local model has been simplified to obtain a more operational layerwise model called M4-5N $[15,16]$. In this model, a polynomial approximation of stresses in each layer is proposed and the laminate is modeled by a superposition of Reissner plates [17] coupled with 
interfacial stresses which ensure the continuity of the stress vector across the interfaces [16]. The M4-5N has already been validated for linear elastic problems [16].

Since layerwise models predict accurately stresses in the layers of a laminate, they are suitable to the prediction of failure onset in laminates. Carrera and Giunta [18] proved that a layerwise model can estimate correctly stress concentrations and predict accurately failure in a multilayered plate. In [19], Nali and Carrera proved the potential of a family of layerwise models to determine the failure envelopes when being applied with suitable failure criteria of composite materials. The case of in-plane biaxial loading was considered. Some of the criteria assayed were the maximum strain, Tsai-Wu [20], Tsai-Hill [21] and LaRCO3 [22] failure criteria. The latter is the most complete since it takes into account most failure phenomena in fibre reinforced unidirectional composites. In [23], Hadj Ahmed et al applied a layerwise model to the probabilistic analysis of failure in double lap adhesively bonded joints in order to determine the optimum length and thickness of the adhesive layer. In [24], Diaz and Caron predicted accurately thickness and ply angle effects on delamination onset in cross-ply carbonepoxy laminates by making use of the M4-5N layerwise model and a criterion involving the interfacial stresses. Dallot and Sab [25] and Limam et al [26] have also proved the potential of layerwise methods in predicting failure with a limit analysis approach.

The failure predictions mentioned above consider linear elastic materials (except for the limit analysis approaches) and perfect interfaces. Nevertheless, non-linear phenomena such as plasticity and ratcheting [27] are usually encountered prior to failure when dealing with adhesives or other polymers. Cognard et al. have carried out experiments with adhesive joints which confirm that significant plastic strains in the adhesive layer precede by far failure initiation and affect the behavior of the joint [28]. A DruckerPrager-type yield function is more suitable than a Von-Mises one for adhesives [29]. In [30], Chataigner et al performed original observations and measurements in a double lap adhesively bonded joint which showed the evolution and growth of a plastic zone in the adhesive layer. In composite laminates, similar non-linear phenomena have also been observed at the interfaces of cross-ply carbon epoxy laminates [31,32]. Diaz and Caron measured an interface sliding which seemed to control a mode III delamination onset at the free edges of the laminates that they tested [31]. This sliding is due to important 
plastic strains within a thin matrix layer located between composite layers. Owing to the experimental results mentioned above, if the adhesive layer or the thin matrix layer is modeled as an interface, this interface should be considered as imperfect and its nonlinear constitutive equations must be deduced from the $3 \mathrm{D}$ plastic equations (yield function and flow rule). In most adhesive joint designs, the thickness of the adhesive layer is less than $10 \%$ of the thickness of the adherends and an interface model for the adhesive is thus preferable in order to reduce the computational cost of the numerical tool derived from the model. Few layerwise models take into account imperfect interfaces and their non-linear constitutive equations. In Part 1 of this work, the layerwise model called M4-5N for the analysis of laminates with imperfect interfaces developed by Aquino de los Rios et al [33] was enhanced to accurately predict stresses, strains and displacement discontinuities across interfaces.

In this paper, the enhanced layerwise model M4-5N developed in Part 1 of this investigation is confronted to experimental measurements. It is worth mentioning that, in the case of adhesively bonded joints, owing to the simplification of the adhesive layer in the layerwise modeling (it is modeled as an imperfect interface), complex 3D experimental phenomena may not be reproduced with our model. For example, if brittle adhesives are used, the fillet radius at the edge of the adhesive layer of the adhesive joint reduces the intensity of the stress singularity and increases the strength of the adhesive joint. In this case, our theoretical approach is not able to take into account the fillet radius in the modeling and a finer model of the adhesive layer would be required. The model is therefore not suitable for failure prediction in adhesive joints exhibiting a high sensitivity of the joint strength to the manufacturing quality of the adhesive application at the edges of the joints. On the one hand, if the fillet radius is not controlled, an important scattering of strength would be expected. On the other hand, controlling the fillet radius may become a complicate task. Thus, this case is not recommended for real world applications. In spite of the above mentioned defects, our modeling is suitable for those robust applications where the geometrical defects of the adhesive layer at the edges do not affect the strength of the adhesive joint. This is the case when a ductile adhesive is employed. Three cases considering such a kind of adhesive are analyzed in this paper and a failure prediction is performed by making use of pertinent criteria. 
This paper is divided in two parts. In the first part, an experimental validation of the model is proposed by making use of the experiments with double lap joints performed by Chataigner et al in [30]. In the second part, suitable failure criteria are applied to predict failure onset in three types of adhesive joints and to compare the predictions to experimental results. The first type of joint is the double lap joint with composite adherends tested by Chataigner et al [30] and exhibiting adherend failure. The second type is a T-peel joint with steel substrates tested by Castagnetti et al in [34] and showing a cohesive failure. The third type is also a T-peel joint but with aluminium adherends tested by Castagnetti et al and exhibiting adhesive failure.

\section{Experimental validation of the model}

In [33], a comparison of the model results with the interlaminar sliding observed by Diaz and Caron [31] at the free edge of cross-ply carbon-epoxy laminates subjected to a tension load was proposed to identify the model parameters and test the accuracy of the model. In these laminates the "interface layer" was a thin matrix layer and its properties appearing in the model were those of an elastic, perfectly plastic material: the Young's modulus, the Poisson's ratio and the yield stress. In these experiments the interlaminar sliding was uniform along the edge of the laminate and was mainly due to interfacial shear stresses. The interface properties were obtained by predicting the interfacial sliding and fitting the plot of the measured sliding against the tensile load. A good prediction of the plot was observed.

In the present paper, more complex experimental observations and measurements in adhesive joints are used to validate the enhanced layerwise model. The experiments carried out by Chataigner et al. in [30] consisted on tensile tests on double lap adhesive joints with an elasto-plastic adhesive exhibiting important plastic strains prior to adherend failure. The UD carbon epoxy adherends were bonded with an epoxy adhesive. The properties of the involved materials are shown in Table 1. In Figure 1, the behavior of the adhesive subjected to a tensile load is shown. Plasticity with hardening is observed. The geometry of the adhesive joints is shown in Figure 2. In order to ease the measurement of the sliding between the inner and outer adherends, vertical lines 
were drawn along the lap length as shown in Figures 2 and 3. As will be shown later, the sliding is not uniform along the adhesive length.

With our layerwise model, owing to its small thickness, the adhesive layer is modeled as an imperfect interface. The predicted displacement discontinuity $\gamma_{1}$ across the imperfect interface is to be compared to the measured sliding between adherends. The stress state at the imperfect interface is deduced from the generalized stresses:

- the in-plane normal stresses are $\sigma_{x x}=\sigma_{1}$ and $\sigma_{y y}=\sigma_{2}$

- the in-plane shear stress is $\sigma_{12}$

- the peel stress is $\sigma_{3}$

- the out-of plane shear stresses $\sigma_{x z}=\tau_{13}$ and $\sigma_{y z}=\tau_{23}$.

These generalized stresses are $z$-independent.

It is well known that the adhesive behavior may exhibit a strong dependence on the hydrostatic stress and that a Drucker-Praguer-like model would predict it accurately. This type of model would require more tests for the parameters identification. For the sake of simplicity, an elastic-plastic model with isotropic hardening is adopted in this paper. The yield function is

$$
f(\boldsymbol{\sigma})=\sigma^{e q}-K p^{a}-\sigma_{0}
$$

where $\sigma^{e q}$ is the equivalent Von-Mises stress in the "interface layer"; $K, a$, and $\sigma_{0}$ are properties of the adhesive which are identified by fitting the experimental curve in Figure 1. After identification we obtain $K=60 \mathrm{MPa}, a=0.2$ and $\sigma_{0}=11 \mathrm{MPa}$. A plane strain state is assumed. The considered geometry and the boundary conditions are shown in Figure 2.

In Figure 4, the experimental and theoretical slidings are plotted against the position $x$ along the lap length for a load of $26 \mathrm{KN}$. It can be observed that the sliding is not uniform along the lap length and the layerwise model provides accurate predictions of the sliding. In Figure 5, the applied load is plotted versus the sliding deduced from line A in Figure 2 (located at $1.2 \mathrm{~mm}$ from the end). One may observe that the sliding at the given point is not proportional to the applied load (plasticity has an important effect). Once again, the layerwise model provides very accurate predictions. 
It is clear that the evaluation of the accuracy of the model in the present paper uses more complex results than those used by Aquino de los Rios et al in [33]. Moreover, in the present paper, the interface properties are obtained from bulk material tests instead of an identification based on fitting the sliding measurements.

\section{Failure prediction}

\subsection{Prediction of adherend failure}

In this subsection, we analyze once again the experimental results obtained by Chataigner et al [30] with double lap joints with an elastoplastic epoxy adhesive. Three adhesive lengths were tested $(62 \mathrm{~mm}, 40 \mathrm{~mm}$ and $20 \mathrm{~mm})$. In these joints, the failure initiation occurred at the inner composite adherends (delaminations within the adherend and near the composite/adhesive interface). Our model reveals that shear and normal stresses that would cause delamination reach their maximum at the vicinity of point $B$ in Figure 2. These stresses and the interfacial stresses at the adherend/adhesive interface are practically the same. Let us now employ a Von-Mises-like criterion involving the out of plane stresses to predict delamination onset within the adherends:

$$
\sqrt{\sigma_{3}^{2}+3 \tau_{13}^{2}}-\sigma^{f}>0
$$

where $\sigma^{f}$ is the out-of plane strength of the adherend. The axial stress that leads to failure onset in the longer specimens is $1700 \mathrm{MPa}$ [30]. The model predicts $57 \mathrm{MPa}$ for the $\sqrt{\sigma_{3}^{2}+3 \tau_{13}^{2}}$ stress. We thus choose $\sigma^{f}=57 \mathrm{MPa}$ in our criterion for predicting failure for any other adhesive length. In Figure 6, the theoretical and experimental critical axial stresses are plotted against the adhesive length. In this Figure 6, we also added the plot of the predictions of Volkersen's theory [35] (a shear-lag model) widely used for adhesive joint design. This theory is based on linear elastic approximations of the stress field. The layerwise model reproduces accurately the experimental curve whereas the Volkersen's theory overestimates considerably the strength for small adhesive lengths. These results prove then that the model is suitable to predict a joint failure when plasticity in the adhesive layer is due predominantly to out of plane shear stresses. 
Let us point out that in this example the failure criterion in the adherend only considered delamination in the composite adherend layers. In a general case for composite adherends, the failure criterion should also be able to predict transverse cracking. The M4-5N model with a pertinent failure criterion offers the possibility to perform this task. For this, a more complete characterization of the adherends is required in order to determine the critical generalized forces and stresses that would appear in the failure criterion.

\subsection{Prediction of cohesive and adhesive failure}

In this subsection, we analyze the experimental results obtained by Castagnetti et al [34] with bonded T-peel joints. Aluminum and steel adherends were tested with a same epoxy adhesive. The geometry of the specimens is shown in Figure 7 and the dimensions and notations of the coupons are detailed in Table 2. Two adherend thicknesses (2 and $3 \mathrm{~mm})$ and two distances $b(12$ and 20mm) of the force axis to the left end of the adhesive were considered (see Figure 7). The adhesive thickness was $s_{a}=0.1 \mathrm{~mm}$ and the specimen width was $25 \mathrm{~mm}$. The elastic properties of the materials involved are shown in Table 3. Five repetitions were performed for each specimen type. The averages of the failure forces (peak forces) measured during the tests are listed in Table 2. Steel specimens exhibited a cohesive failure whereas aluminum specimens showed an adhesive failure.

In order to apply our layerwise model to the prediction of failure in these joints, symmetry is applied as shown in Figure 7 (left part). At the left end of the adherend, a force $F$ and a bending moment $M=b \times F$ are applied. A plane strain state is assumed. In [34], Castagnetti et al. assumed a purely brittle response for the adhesive and didn't characterize its real mechanical behavior by means of a tensile test. In [36], Goglio et al. performed mechanical tests with bulk specimens of the same adhesive tested by Castagnetti et al. A quick analysis of experimental stress-strain curves during the quasistatic tests made in [36] proves that an elastic perfectly plastic model and a 34MPa yield stress are a reasonable choice for modeling the behavior of the adhesive. 
To predict a cohesive failure, a criterion based on a maximum cumulative plastic strain is adopted. When the failure force is applied to steel specimens, the average of predicted maximum cumulative plastic strains $p$ among the specimens is 0.0541 . These maximum values are reached at the left edge of the joint. The cohesive failure criterion is then: $p>0.0541$. With this criterion, the M4-5N model predicts the failure loads for each specimen. Figure 8, displays the plot of the experimental and theoretical failure forces. The M4-5N predictions are very accurate.

To predict an adhesive failure, we adopt a criterion similar to that obtained by HartSmith [10] to predict delamination onset based on a maximum "interfacial strain". When the failure force is applied to aluminum joint specimens, the average of predicted maximum interfacial opening displacements $\gamma_{3}$ among the specimens is $1.63 \mu \mathrm{m}$ at the left edge of the interface. The adhesive failure criterion is then: $\varepsilon_{z}=\frac{\gamma_{3}}{s_{a}}>0.0163$, where $\varepsilon_{z}$ is the interfacial opening strain. With this criterion, the M4-5N model predicts the failure loads for each specimen. Let us point out that the M4-5N model predicts that the critical strain is reached without plasticity onset (the yield stress is not attained prior to the failure of aluminum joint specimens). Figure 9, displays the plot of the experimental and theoretical failure forces. The M4-5N predictions are correct but not as accurate as those for steel T-joint specimens. Let us remark that an important scattering of experimental data exists. This scattering is surely due to manufacturing defects and the sensitivity of the joint strength to the quality of the adhesive left edge as suggested by Castagnetti et al [34]. The important sensitivity of the joint strength to the manufacturing quality is a proof that failure occurs with negligible plastic strains in the adhesive which may smooth the stress concentrations. In this manner, the zero plastic strains calculated by the M4-5N model in the considered joints agree with the previous reasoning.

The above examples prove then that the model is also suitable to predict adhesive and cohesive failures due to peel stresses.

\section{Conclusion}


In this paper, an experimental validation of an enhanced layerwise model for laminates with imperfect interfaces was performed. In this validation, the measurements of plastic slidings in a double-lap adhesively bonded joint [30] were accurately predicted by the model. The model was then applied to the failure prediction in double-lap and T-peel joints. Pertinent failure criteria involving the plastic strains in the adhesive, the stresses in the adherends and the total strains in the adhesive helped to predict accurately cohesive, adherend and adhesive failures, respectively.

The enhanced layerwise model and pertinent failure criteria prove to be a suitable tool for the analysis and design of adhesive joints with a plate topology and thin layers of an elastic-plastic adhesive. Owing to its accuracy and less computational cost, this layerwise model is an attractive alternative to solid finite element calculations. In a subsequent paper, the limits in the modeling of adhesive layers as imperfect interfaces will be analyzed. This task will enable the determination of a maximum ratio of adhesive thickness to adherend thickness for an accurate and reliable modeling.

\section{Acknowledgements}

The authors wish to acknowledge CONACYT (grant no. CB-2007-01 79467 002) and US AIR FORCE (award no. FA9550-09-1-0394) for their financial support.

\section{References}

[1] Ferreira AJM, Carrera E, Cinefra M, Roque CMC, Polit O. Analysis of laminated shells by a sinusoidal shear deformation theory and radial basis functions collocation, accounting for through-the-thickness deformations. Composites Part B: Engineering 2011;42(5):1276-1284.

[2] Hadj-Ahmed R, Foret G, Ehrlacher A. Stress analysis in adhesive joints with a multiparticle model of multilayered materials (M4). International Journal of Adhesion and Adhesives 2001;21(4):297-307.

[3] $\mathrm{Wu} \mathrm{X-F}$, Jenson RA. Stress-function variational method for stress analysis of bonded joints under mechanical and thermal loads. International Journal of Engineering Science 2011;49(3):279-294. 
[4] Nemeş O, Lachaud F. Double-lap adhesive bonded-joints assemblies modeling. International Journal of Adhesion and Adhesives 2010;30(5):288-297.

[5] Allix O, Lévêque D, Perret L. Identification and forecast of delamination in composite laminates by an interlaminar interface model. Composites Science and Technology 1998;58(5):671-678.

[6] Brewer JC, Lagace PA. Quadratic stress criterion for initiation of delamination. Journal of Composite Materials 1988;22:1141-1155.

[7] Kim RY, Soni SR, Experimental and analytical studies on the onset of delamination in laminated composites. J Compos Mater, 18 (1984), pp. 70-80.

[8] Da Silva LFM, Adams RD. Techniques to reduce the peel stresses in adhesive joints with composites. International Journal of Adhesion and Adhesives 2007;27(3):227-235.

[9] Crocombe AD, Bigwood DA, Richardson G. Analysing structural adhesive joints for failure. International Journal of Adhesion and Adhesives 1990;10(3):167-178.

[10] Hart-Smith LJ. Adhesive bonded double lap joints. Technical Report NASA-CR112235, NASA-Langley Contract Report; 1973.

[11] Carrera E. Developments, ideas, and evaluations based upon Reissner's mixed variational theorem in the modelling of multilayered plates and shells. Applied Mechanical Reviews 2001;54(4)301-329.

[12] Carrera E. Theories and finite elements for multilayered plates and shells: a unified compact formulation with numerical assessment and benchmarking. Archives of Computational Methods in Engineering 2003;10(3):215-296.

[13] Reissner E. On a variational theorem in elasticity. Journal of Mathematics and Physics 1950;29:90-95.

[14] Pagano NJ. Stress fields in composite laminates. International Journal of Solids and Structures 1978;14(5):385-400.

[15] Chabot A. Analyse des efforts à l'interface entre les couches des matériaux composites à l'aide des M4. Doctoral dissertation, ENPC, France, 1997.

[16] Carreira RP, Caron JF, Diaz Diaz A. Model of multilayered materials for interface stresses estimation and validation by finite element calculations. Mechanics of Materials 2002;34(4):217-230.

[17] Reissner E. The effect of transverse shear deformation on the bending of elastic plates. Journal of Applied Mechanics 1945;A:69-77. 
[18] Carrera E, Giunta G. Hierarchical models for failure analysis of plates bent by distributed and localized transverse loadings. Journal of Zhejiang University Science A 2008;9(5):600-613.

[19] Nali P, Carrera E. A numerical assessment on two-dimensional failure criteria for composite layered structures. Composites Part B: Engineering 2011. doi:10.1016/j.compositesb.2011.06.018

[20] Tsai SW, Wu EM. A general theory of strength for anisotropic materials. Journal of Composite Materials 1971;5(1) (1971):58-80.

[21] Tsai SW. Strength theories of filamentary structures fundamental aspects of fibre reinforced plastic composites. WileyInterscience, New York (1968).

[22] Dávila CG, Camanho PP. Failure criteria for frp laminates in plane stress. Tech. Rep. NASA/TM-2003-212663; 2003..

[23] Hadj Ahmed R, Foret G, Ehrlacher A. Probabilistic analysis of failure in adhesive bonded joints. Mechanics of Materials 2001;33(2):77-84.

[24] Diaz Diaz A, Caron JF. Prediction of the onset of mode III delamination in carbonepoxy laminates. Composite Structures 2006;72(4):438-445.

[25] Dallot J, Sab K. Limit analysis of multi-layered plates. Part II: Shear effects. Journal of the Mechanics and Physics of Solids 2008;56(2):581-612.

[26] Limam O, Foret G, Ehrlacher A. RC two-way slabs strengthened with CFRP strips: experimental study and a limit analysis approach. Composite Structures 2003;60(4):467-471.

[27] Lin YC, Chen XM, Zhang J. Uniaxial ratchetting behavior of anisotropic conductive adhesive film under cyclic tension. Polymer Testing 2011;30(1):8-15.

[28] Cognard JY, Davies P, Sohier L, Créac'hcadec R. A study of the non-linear behaviour of adhesively-bonded composite assemblies. Composite Structures 2006;76(1-2):34-46.

[29] Wang CH, Chalkley P. Plastic yielding of a film adhesive under multiaxial stresses. International Journal of Adhesion and Adhesives 2000;20(2):155-164.

[30] Chataigner S, Caron JF, Duong VA, Diaz Diaz A. Experimental and numerical investigation of shear strain along an elasto-plastic bonded lap joint. Construction and Building Materials 2011;25(2):432-441.

[31] Diaz Diaz A, Caron JF. Interface plasticity and delamination onset prediction. Mechanics of Materials 2006;38(7):648-663. 
[32] Lecomte-Grosbras P, Paluch B, Brieu M, De Saxcé G, Sabatier L. Interlaminar shear strain measurement on angle-ply laminate free edge using digital image correlation.

[33] Aquino de Los Rios GS, Castañeda Balderas R, Duong VA, Chataigner S, Caron JF, Ehrlacher A, Foret G, Diaz Diaz A, Laminated materials with plastic interfaces: modeling and calculation. Modeling and Simulation in Materials Science and Engineering 2009;17(2):1-21.

[34] D. Castagnetti, E. Dragoni, A. Spaggiari. Failure analysis of bonded T-peel joints: Efficient modelling by standard finite elements with experimental validation. International Journal of Adhesion and Adhesives 2010;30(5):306-312.

[35] Volkersen O. Die nietkraftverteilung in zugbeanspruchten mit konstanten laschenquerschritten. Luftfahrtforschung 1938;15:41-47.

[36] Goglio L, Peroni L, Peroni M, Rossetto M. High strain-rate compression and tension behaviour of an epoxy bi-component adhesive. International Journal of Adhesion and Adhesives 2008;28(7):329-339. 
Figure 1. Stress strain curve of the elasto-plastic adhesive [30].

Figure 2. Geometry of the double lap joint and boundary conditions.

Figure 3. Measuring the sliding between adherends [30].

Figure 4. Slidings along the lap length (experiments [30] and predictions).

Figure 5. Applied force vs. slidings on line A in Figure 2 (experiments [30] and predictions).

Figure 6. Critical stress vs. lap length (experiments [30] and predictions).

Figure 7. T-peel joints: geometry [34] and modeling (dimensions are in mm).

Figure 8. Failure loads for steel/epoxy specimens (experiments [34] and predictions).

Figure 9. Failure loads for aluminum/epoxy specimens (experiments [34] and predictions). 


\begin{tabular}{c|c|c}
\hline Property $\backslash$ Material & Adhesive & Adherend \\
\hline Young's modulus $(\mathrm{GPa})$ & 2.5 & $\begin{array}{c}162 \text { (fibre direction) } \\
10.6 \text { (transverse direction) }\end{array}$ \\
\hline Poisson's ratio & 0.3 & 0.3 \\
\hline Out-of- plane shear modulus $(\mathrm{GPa})$ & & 4.1
\end{tabular}

Table 1. Elastic properties of involved materials [30].

\begin{tabular}{|c|c|c|c|c|c|c|c|c|}
\hline Adherend & \multicolumn{4}{|c|}{ Steel } & \multicolumn{4}{c|}{ Aluminum } \\
\hline Specimen & $1 \mathrm{~s}$ & $2 \mathrm{~s}$ & $3 \mathrm{~s}$ & $4 \mathrm{~s}$ & $1 \mathrm{a}$ & $2 \mathrm{a}$ & $3 \mathrm{a}$ & $4 \mathrm{a}$ \\
$t(\mathrm{~mm})$ & 2 & 3 & 2 & 3 & 2 & 3 & 2 & 3 \\
$b(\mathrm{~mm})$ & 20 & 20 & 12 & 12 & 20 & 20 & 12 & 12 \\
$F(\mathrm{~N})$ & 487.5 & 779.2 & 794.5 & 1289.3 & 65.3 & 88.6 & 102.7 & 129.6 \\
\hline
\end{tabular}

Table 2. Specimen notation, dimensions and failure forces [34].

\begin{tabular}{|c|c|c|c|}
\cline { 2 - 4 } \multicolumn{1}{c|}{} & Steel & Aluminum & Adhesive \\
\hline Young's modulus (GPa) & 206 & 69 & 1.7 \\
Poisson's ratio & 0.3 & 0.3 & 0.3 \\
\hline
\end{tabular}

Table 3. Elastic properties of the materials applied in the T-joints [34]. 